\title{
EFFECT OF VARIOUS TYPES AND RATES OF ORGANIC MANURES ON VEGETATIVE GROWTH, PIGMENTS AND CHEMICAL COMPOSITION OF LEAVES, YIELD AND SPEAR PIGMENTS OF BROCCOLI. \\ Hossein, H. A. \\ Hort. Dept., Fac. of Agric., Suez Canal University, Ismailia, Egypt
}

\begin{abstract}
Two Field experiments were conducted at the Experimental Farm of the Faculty of Agriculture, Suez Canal university during the winter seasons of 2004-2005 and 2005-2006 to study the effect of three types of organic manures, i.e poultry manure, cattle manure and sheep manure in comparison with conventional fertilization (cattle manure + mineral NPK) on broccoli vegetative growth, leaves chemical composition, leaf pigments, yield and its components and spear pigments. The organic manures and conventional fertilization (cattle manure + mineral nitrogen fertilizer) were applied according to their content of nitrogen at three rates: 100, 140 and $180 \mathrm{~kg} \mathrm{~N} /$ feddan.

The obtained results indicated that conventional fertilization gave the highest values of stem length; stem diameter; stem weight; number of leaves per plant; both fresh and dry weights of leaves; chlorophyll a and b, total chlorophyll and carotenoids contents of leaves, N, P and K contents of leaves; main spear diameter; main spear weight; main spear yield (Ton/Fed); average side spear diameter; number of side spear per plant; side spears yield per plant; side spears yield (Ton/Fed); total yield; chlorophyll a, chlorophyll $b$, total chlorophyll and carotenoids contents of main spear. The second fertilizer with positive increase in the previous parameters was poultry manure followed by cattle manure then sheep manure. The previous parameters except number of leaves per plant were increased with increasing the rate of manure application providing $\mathrm{N}$-level from 100 to $180 \mathrm{Kg} / \mathrm{Fed}$. The conventional fertilization containing $180 \mathrm{~kg} \mathrm{~N} /$ Fed recorded the highest values of stem length; stem diameter; stem weight; number of leaves per plant; both fresh and dry weights of leaves; chlorophyll a, chlorophyll b, total chlorophyll and carotenoid, contents of leaves; N, P, and $\mathrm{K}$ contents of leaves and chlorophyll $\mathrm{a}$, chlorophyll $\mathrm{b}$, total chlorophyll and carotenoids of main spear, while conventional fertilization containing $140 \mathrm{~kg} \mathrm{~N} / \mathrm{Fed}$ gave the highest values of yield and its components.
\end{abstract}

\section{INTRODUCTION}

Broccoli (Brassica olerace var. italica) is popular vegetable around the world especially in Europe and USA. It is grown in Egypt recently as a new vegetable crop in winter season, but in very limited areas. Broccoli spears is rich in vitamin c. vitamin a, calcium, iron, riboflavin, niacin and protein. Addition of organic manures in sandy soils can increase nutrients availability, cation exchange capacity and micro nutrients. They contain high organic matter, improve drought tolerance (Hotlink et al., 1991) and increases soil microbial activities (Meissner-Smejkal, 2000). Fisher and Richter (1984), Borin et al. (1987) and Browaldh (1992) reported that organic manure usage lead to produce clean plant product. Also, organic manures do not cause environmental pollution compared with chemical fertilizers. Moreover, the 
Hossein, H. A.

increased demand for organic products has also been an incentive for producers in sub-tropical countries to start organic production for export. Some studies were conducted to compare effect of mineral or conventional fertilization with effect of organic manure on other vegetable crops. The results of Gliesman et al. (1996) on strawberry and Hossein (2002) on onion, showed that vegetative growth and yield was decreased in organic production as compared with under conventional system.

With concern to the effect of organic manure rate on growth and yield of vegetable crops El-Sheikh and Salam (1997) showed that increasing rate of chicken manure significantly increased vegetative growth, fruit number per plant, number of trusses per plant yield and fruit components of tomato. Furthermore Ali et al. (2001) reported that increasing the rate of chicken manure recorded an increase in plant height, number and area of leaves, fresh dry weight of whole plant, and its leaves and yield of garlic.

Previous investigations on the effects of organic manures in comparison with conventional fertilization on growth and yield of broccoli plants are limited. Therefore, this study was conducted to test the effects of different manures and conventional fertilization at three $\mathrm{N}$ levels on the growth and yield of broccoli.

\section{MATERIALS AND METHODS}

Two field experiments were carried out during the winter seasons of 2004-2005 and 2005-2006 at the Experimental Research Farm, Faculty of Agriculture, Suez Canal University, Ismailia Governorate to study the effect of various types of organic manures i.e. poultry manure, cattle manure and sheep manure, and conventional Fertilization at three $\mathrm{N}$ levels (100, 140 and $180 \mathrm{~kg} \mathrm{~N} / \mathrm{Fed}$ ) on vegetative growth, leaves chemical composition, leaf pigments, yield and its components and spear pigments of broccoli cv. Southern Star. The fertilizers (organic manures or conventional Fertilization) were applied according to their contents of nitrogen. Seeds were sown in seedling trays containing peat moss and vermiculite (1: 1) (by volume) on 20 and 23 September in the first and second season, respectively. The transplants were raised in the trays for one month, then transplanted in the field.

The physical and chemical properties of the experimental soil were: 96.1 and 95.82 sand, 2.85 and 2.71 silt, 1.10 and 1.48 clay, 8.11 and 8.24 $\mathrm{PH}, 1.67$ and $1.89 \mathrm{~mm}$ hose $/ \mathrm{cm}, 0.73$ and $0.73 \mathrm{OC}, 3.87$ and 3.99 available $\mathrm{N}, 3.00$ and $3.11 \mathrm{P}$ and 10.51 and $15.58 \mathrm{~K}$. The experimental design was spilt- plot with three blocks. The main plots were presented as type of fertilizer, while $\mathrm{N}$ level, were distributed as sub-plots. The sub-plot area was $14 \mathrm{~m}^{3}$ which contained 4 rows each $5 \mathrm{~m}$ long and $0.7 \mathrm{~m}$ wide. The distance between plants was $0.5 \mathrm{~m}$. The seedlings were transplanted on one side of row.

Chemical analysis of the used organic manure is shown in Table (1). 
Table (1): Chemical analysis and the application rate of the organic manures used during 2004-2005 and 2005-2006 seasons.

\begin{tabular}{|c|c|c|c|c|c|c|}
\hline \multirow[b]{2}{*}{$\begin{array}{l}\text { Organic manure } \\
\text { character }\end{array}$} & \multicolumn{2}{|c|}{ Poultry manure } & \multicolumn{2}{|c|}{ Cattle manure } & \multicolumn{2}{|c|}{ Sheep manure } \\
\hline & $\begin{array}{l}\text { 2004- } \\
2005\end{array}$ & $\begin{array}{l}2005- \\
2006\end{array}$ & $\begin{array}{l}\text { 2004- } \\
2005\end{array}$ & $\begin{array}{l}2005- \\
2006\end{array}$ & $\begin{array}{l}2004- \\
2005\end{array}$ & $\begin{array}{l}2005- \\
2006\end{array}$ \\
\hline Weight of $\mathrm{m}^{3}(\mathrm{~kg})$ & 432 & 453 & 530 & 541 & 561 & 570 \\
\hline Moisture percentage & 55 & 53 & 42 & 46 & 42 & 40 \\
\hline PH & 6.2 & 6.3 & 7.3 & 7.0 & 6.5 & 6.6 \\
\hline Ec (mm hmose/cm) & 4.8 & 4.6 & 13.9 & 13.8 & 7.1 & 7.1 \\
\hline Organic carbon \% & 35.5 & 29.1 & 27.3 & 29.2 & 14.4 & 15.6 \\
\hline Organic matter & 70.2 & 64.9 & 26.4 & 28.2 & 65.4 & 61.9 \\
\hline Total nitrogen $\%$ & 2.94 & 2.85 & 1.13 & 1.20 & 2.08 & 1.99 \\
\hline $\mathbf{C} / \mathbf{N}$ ratio (\%) & $10.37: 1$ & $10.21: 1$ & $24.15: 1$ & $24.33: 1$ & $6.29: 1$ & $7.84: 1$ \\
\hline Total phosphorus \% & 0.68 & 0.75 & 0.55 & 0.51 & 0.69 & 0.73 \\
\hline Total potassium \% & 2.1 & 1.9 & 1.7 & 1.9 & 1.74 & 1.64 \\
\hline Ferrous mg/kg & 180 & 193 & 799 & 780 & 805 & 811 \\
\hline Manganese $\mathrm{mg} / \mathrm{kg}$ & 215 & 190 & 133 & 142 & 93 & 95 \\
\hline Copper mg/kg & 40 & 43 & 20 & 24 & 50 & 52 \\
\hline Zinc $\mathbf{~ g ~} / \mathbf{k g}$ & 105 & 111 & 80 & 79 & 100 & 104 \\
\hline $\begin{array}{l}\text { Application rate } \\
(\mathrm{kg})=100 \mathrm{~kg} \mathrm{~N} / \mathrm{Fed}\end{array}$ & 5535 & 5636 & 13194 & 12774 & 7168 & 7386 \\
\hline $\begin{array}{l}\text { Application rate } \\
(\mathrm{kg})=140 \mathrm{~kg} \mathrm{~N} / \mathrm{Fed}\end{array}$ & 7750 & 7891 & 17947 & 17133 & 10835 & 10341 \\
\hline $\begin{array}{l}\text { Application rate } \\
(\mathrm{kg})=180 \mathrm{~kg} \mathrm{~N} / \mathrm{Fed}\end{array}$ & 8657 & 9018 & 21111 & 19466 & 11469 & 11819 \\
\hline
\end{tabular}

In both seasons, the experiment included 12 treatments as follows:

$$
\text { Fertilizer }
$$

$N$ level

Mineral N.P and K Fertilizers + cattle manure $\left(15 \mathrm{~m}^{3} / \mathrm{Fed}\right)$

Mineral N.P and K Fertilizers + cattle manure $\left(15 \mathrm{~m}^{3} / \mathrm{Fed}\right)$

Mineral N.P and K Fertilizers+ cattle manure $\left(15 \mathrm{~m}^{3} / \mathrm{Fed}\right)$

$100 \mathrm{~kg} / \mathrm{Fed}$

$140 \mathrm{~kg} / \mathrm{Fed}$

$180 \mathrm{~kg} / \mathrm{Fed}$

Poultry manure

Poultry manure

Poultry manure

$100 \mathrm{~kg} / \mathrm{Fed}$

$140 \mathrm{~kg} / \mathrm{Fed}$

$180 \mathrm{~kg} / \mathrm{Fed}$

cattle manure

$100 \mathrm{~kg} / \mathrm{Fed}$

$140 \mathrm{~kg} / \mathrm{Fed}$

cattle manure

$180 \mathrm{~kg} / \mathrm{Fed}$

$100 \mathrm{~kg} / \mathrm{Fed}$

Sheep manure

Sheep manure

Sheep manure

$140 \mathrm{~kg} / \mathrm{Fed}$

$180 \mathrm{~kg} / \mathrm{Fed}$

Ammonium sulphate $(20.5 \% \mathrm{~N})$ was used as a source of mineral nitrogen and potassium sulphate was applied at the rate of $75 \mathrm{~kg} /$ Feddan. Both ammonium sulphate and potassium sulphate were added after 2, 4, 6 and 8 weeks from transplanting. Phosphorus was added in the form of calcium superphosphate $\left(15.5 \mathrm{P}_{2} \mathrm{O}_{5}\right)$ at the rate of $200 \mathrm{~kg} /$ Feddan. Calcium superphosphate and organic manures were added at the time of soil preparation. Furrow irrigation was applied every three days. The other normal agricultural treatments for broccoli plants were practiced. 
Hossein, H. A.

Data Recorded:

The following data were recorded during the plant growth period and harvesting period.

1- Vegetative growth characters:

Five plants from each sub-plot were randomly taken at 100 days after transplanting and the following data were recorded. Stem length, stem diameter, stem weight, number of leaves per plant, fresh weight of leaves and dry weight of leaves after drying in an oven for $72 \mathrm{hrs}$.

2- Leaf pigments

Disks sample from the third lower leaves were taken from each sub- plot at 80 days after transplanting to determine chlorophyll a, chlorophyll $b$ and total chlorophyll as well as carotenoids according to the methods described by Wettestein (1957).

\section{3- Leaves chemical composition}

The dried leaves were finely ground and digested and total nitrogen was determined according to Bremner and Mulvaney (1992). Phosphorus was estimated colorimectrically according to Olesen and Sommers (1982). Potassium was also determined flame photometrically due to the methods described by Jackson (1970).

\section{4- Yield and its components:}

\section{a- Main Spears Characters}

Five main spears were cut from each sub-plot when main spears were judged to be mature then the following characters were determined:

a-1- Main spear diameter $(\mathrm{cm})$.

a-2- Main spear weight.

\section{b- Side spears characters}

b-1- Average side spear diameter.

b-2- Number of side spear plant.

b-3- Side spears yield per plant.

\section{c- Yield}

c-1- Main spears yield.

c-2- Side spears yield.

c-3- Total yield.

\section{5- Main spears pigments}

Spears samples from each sub plot were taken during maturity stage to determine chlorophyll a, chlorophyll $b$ and total chlorophyll as well as carotenoids according the methods described by Wettestein (1957).

\section{Data Statistical Analysis:}

All obtained data were subjected to analysis of variance according to Sendecor and Cochran (1980) and the least significant difference (LSD) was calculated as mentioned by Gomez and Gomez (1984) at 5\% level.

\section{RESULTS AND DISCUSSION}

\section{1- Vegetative Growth Characters:}

a) Main effect of fertilizer type

Data in Tables (2 and 4) show that conventional fertilization significantly recorded the highest values of stem length, stem diameter, stem 
weight, number of leaves per plant and both fresh and dry weight of leaves in both growing seasons. The second fertilizer with positive increase in the previous parameters was poultry manure, followed by cattle manure then sheep manure. The obtained results are in harmony with those of Gliesman et al. (1996) on strawberry, and Hossein (2002) on onion, where they reported that plant vegetative growth was decreases in the organic production compared with under conventional system. Moreover, the obtained results are in harmony with those of Abde El-Mouty et al. (2000) on squash and El-Mansi et al. (2004) on pea, where they found that chicken manure gave better plant growth expressed as length of plant and number of leaves per plant than cattle manure.

The observed enhancement effect on plant growth with conventional fertilization compared with organic manures may be due to rapid availability of nutrients from mineral fertilizers, for broccoli plant growth. The improvement of vegetative growth with poultry manure as compared with the other organic manure may be inputed to rapid decomposition of poultry manure throughout broccoli growth period.

\section{b- Main effect of $\mathbf{N}$ level}

Data in Tables (2 and 4) indicated that all vegetative growth parameters except number of leaves per plant were significantly increased with increasing $\mathrm{N}$ level from 100 to $180 \mathrm{~kg} /$ Feddan in both seasons.

Table (2): Main effect of fertilizer type and $\mathrm{N}$ level on broccoli vegetative growth during $2004-2005$ season.

\begin{tabular}{|c|c|c|c|c|c|c|}
\hline Character & $\begin{array}{c}\text { Stem } \\
\text { length } \\
(\mathrm{cm})\end{array}$ & $\begin{array}{c}\text { Stem } \\
\text { diameter } \\
(\mathbf{c m})\end{array}$ & $\begin{array}{c}\text { Stem } \\
\text { weight } \\
\text { (g) }\end{array}$ & $\begin{array}{l}\text { No. of } \\
\text { leaves per } \\
\text { plant }\end{array}$ & $\begin{array}{l}\text { Fresh } \\
\text { weight of } \\
\text { leaves } \\
\text { (g) }\end{array}$ & $\begin{array}{l}\text { Dry } \\
\text { weight of } \\
\text { leaves } \\
\text { (g) }\end{array}$ \\
\hline \multicolumn{7}{|l|}{ Fertilizer } \\
\hline $\begin{array}{l}\text { Mineral NPK } \\
\text { cattle manure }\end{array}$ & +13.03 & 3.55 & 119.15 & 17.05 & 488.28 & 76.317 \\
\hline Poultry manure & 12.01 & 2.86 & 96.14 & 13.92 & 245.50 & 45.353 \\
\hline Cattle manure & 8.71 & 2.58 & 55.10 & 11.73 & 160.24 & 29.29 \\
\hline Sheep manure & 6.94 & 1.69 & 46.64 & 9.77 & 97.03 & 16.81 \\
\hline $\begin{array}{c}\text { LSD } 5 \% \\
\mathrm{~N} \text { level }(\mathrm{kg} / \mathrm{Fed})\end{array}$ & 0.77 & 0.19 & 6.78 & 1.64 & 23.27 & 4.22 \\
\hline 100 & 8.913 & 2.23 & 69.77 & 12.18 & 213.91 & 37.27 \\
\hline 140 & 10.21 & 2.65 & 81.53 & 13.20 & 243.00 & 40.42 \\
\hline 180 & 11.39 & 3.13 & 86.47 & 13.95 & 286.38 & 48.14 \\
\hline LSD $5 \%$ & 0.99 & 0.29 & 10.08 & NS & 31.43 & 5.60 \\
\hline
\end{tabular}

The obtained results are in harmony with those of Thakur et al. (1999) on cauliflower and Brahmo et al. (2000) on Broccoli. Moreover, similar results were obtained by Singh and Akhilesh-Singh (2002) on broccoli, Jana and Mukhopadhyay (2007) on cauliflower and Sharma et al. (2002) on broccoli concerning plant height.

The increasing in all vegetative growth parameters of broccoli plants with increasing $\mathrm{N}$ level may be due to nitrogen element being required to form amino acids, amides, peptide and high molecular-weight peptides such as 
Hossein, H. A.

protein (Marschner, 1988). An increase in protein content of vegetables is often positively correlated with increase in content of vitamins such as riboflavin, thiamin and nicotine acid (Dressel and Jung, 1979). Also, nitrogen is necessary for formation of treptophan, a precursor for indole acetic acid (Skoog, 1979).

c- Effect of interaction between fertilizer type and $\mathrm{N}$ level

Data in Tables (3 and 5) illustrate that conventional fertilization containing $180 \mathrm{~kg} \mathrm{~N} /$ Feddan gave significantly the highest values of stem length, stem diameter, stem weight, number of leaves per plant and both fresh and dry weights of leaves in both seasons.

Table (3): Effect of interaction between fertilizer type and $\mathbf{N}$ level on broccoli vegetative growth during 2004-2005 season.

\begin{tabular}{|c|c|c|c|c|c|c|c|}
\hline Treatment & racter & $\begin{array}{l}\text { Stem } \\
\text { length } \\
\text { (cm) }\end{array}$ & $\begin{array}{c}\text { Stem } \\
\text { diameter } \\
(\mathrm{cm})\end{array}$ & $\begin{array}{c}\text { Stem } \\
\text { weight } \\
\text { (g) }\end{array}$ & $\begin{array}{c}\text { No. of } \\
\text { leaves } \\
\text { per plant }\end{array}$ & $\begin{array}{l}\text { Fresh weight of } \\
\text { leaves } \\
(\mathrm{g})\end{array}$ & $\begin{array}{c}\text { Dry weight } \\
\text { of leaves } \\
\text { (g) }\end{array}$ \\
\hline Mineral & 100 & 11.17 & 3.09 & 109.49 & 15.37 & 399.10 & 68.62 \\
\hline NPK +cattle & 140 & 12.55 & 3.75 & 119.31 & 17.62 & 480.79 & 78.77 \\
\hline manure & 180 & 15.38 & 4.17 & 128.64 & 18.15 & 578.28 & 81.56 \\
\hline Poultry & 100 & 10.00 & 2.15 & 72.14 & 13.07 & 214.40 & 41.13 \\
\hline manure & 140 & 12.62 & 2.85 & 105.07 & 14.11 & 236.11 & 37.82 \\
\hline & 180 & 13.40 & 3.57 & 110.53 & 14.58 & 286.00 & 57.11 \\
\hline Cattle & 100 & 8.13 & 2.11 & 51.18 & 11.31 & 150.83 & 25.83 \\
\hline manure & 140 & 8.77 & 2.66 & 55.63 & 11.82 & 159.74 & 28.62 \\
\hline & 180 & 9.23 & 2.95 & 58.48 & 12.06 & 170.15 & 33.42 \\
\hline Sheep & 100 & 6.35 & 1.58 & 45.60 & 8.97 & 84.66 & 13.50 \\
\hline manure & 140 & 6.91 & 1.66 & 46.11 & 9.56 & 95.34 & 16.46 \\
\hline & 180 & 7.55 & 1.84 & 48.22 & 11.00 & 111.08 & 20.47 \\
\hline \multicolumn{2}{|c|}{ LSD $5 \%$} & 1.20 & 0.57 & 20.16 & 3.88 & 62.84 & 11.20 \\
\hline
\end{tabular}

Table (4): Main effect of fertilizer type and $\mathrm{N}$ level on broccoli vegetative growth during $2005-2006$ season

\begin{tabular}{|c|c|c|c|c|c|c|}
\hline Character & $\begin{array}{l}\text { Stem } \\
\text { length } \\
(\mathrm{cm})\end{array}$ & $\begin{array}{c}\text { Stem } \\
\text { diameter } \\
(\mathrm{cm})\end{array}$ & $\begin{array}{l}\text { Stem } \\
\text { weight } \\
\text { (g) }\end{array}$ & $\begin{array}{c}\text { No. of } \\
\text { leaves per } \\
\text { plant }\end{array}$ & $\begin{array}{c}\begin{array}{c}\text { Fresh } \\
\text { weight of } \\
\text { leaves }(\mathrm{g})\end{array} \\
\end{array}$ & $\begin{array}{c}\text { Dry weight } \\
\text { of leaves } \\
\text { (g) }\end{array}$ \\
\hline \\
\hline $\begin{array}{l}\text { Mineral } \\
\text { NPK + cattle } \\
\text { manure }\end{array}$ & 11.08 & 3.93 & 92.69 & 13.39 & 378.95 & 58.83 \\
\hline Poultry manure & 10.82 & 2.67 & 76.63 & 12.55 & 290.68 & 56.67 \\
\hline Cattle manure & 8.51 & 2.41 & 57.87 & 11.75 & 165.39 & 30.31 \\
\hline Sheep manure & 6.49 & 1.81 & 37.83 & 10.20 & 20.31 & 12.18 \\
\hline LSD 5\% & 0.77 & 0.15 & 6.50 & 1.54 & 27.75 & 2.60 \\
\hline $\mathbf{N}$ level (kg/Fed) & & & & & & \\
\hline 100 & 8.25 & 3.25 & 58.93 & 10.76 & & 35.82 \\
\hline 140 & 9.18 & 2.84 & 65.43 & 12.08 & 216.81 & 38.89 \\
\hline 180 & 10.25 & 2.80 & 74.41 & 13.08 & 264.50 & 44.59 \\
\hline LSD 5 & 0.99 & 2.00 & 7.71 & NS & 32.07 & 5.35 \\
\hline
\end{tabular}


Table (5): Effect of interaction between fertilizer type and $\mathrm{N}$ level on broccoli vegetative growth during 2005-2006 season

\begin{tabular}{|c|c|c|c|c|c|c|c|}
\hline Treatment & racter & $\begin{array}{c}\text { Stem } \\
\text { length } \\
\text { (cm) }\end{array}$ & $\begin{array}{c}\text { Stem } \\
\text { diameter } \\
(\mathrm{cm})\end{array}$ & $\begin{array}{c}\text { Stem } \\
\text { weight } \\
\text { (g) }\end{array}$ & $\begin{array}{c}\text { No. of } \\
\text { leaves per } \\
\text { plant }\end{array}$ & $\begin{array}{c}\text { Fresh } \\
\text { weight of } \\
\text { leaves (g) }\end{array}$ & $\begin{array}{c}\text { Dry weight } \\
\text { of leaves } \\
\text { (g) }\end{array}$ \\
\hline Mineral & 100 & 9.41 & 2.89 & 76.65 & 12.21 & 324.05 & 55.08 \\
\hline NPK + cattle & 140 & 10.53 & 3.2 & 89.17 & 13.46 & 350.12 & 56.84 \\
\hline manure & 180 & 13.30 & 3.6 & 112.25 & 14.49 & 462.67 & 64.91 \\
\hline Poultry & 100 & 9.45 & 2.44 & 69.33 & 11.16 & 257.88 & 49.42 \\
\hline manure & 140 & 11.23 & 2.61 & 77.06 & 12.82 & 291.50 & 58.20 \\
\hline & 180 & 11.78 & 2.95 & 83.51 & 13.66 & 319.34 & 62.39 \\
\hline Cattle & 100 & 8.14 & 2.00 & 54.78 & 1017 & 143.12 & 24.78 \\
\hline manure & 140 & 8.52 & 2.51 & 58.73 & 12.23 & 154.49 & 28.41 \\
\hline & 180 & 8.88 & 2.73 & 60.11 & 12.86 & 198.55 & 37.74 \\
\hline Sheep & 100 & 6.01 & 1.65 & 35.03 & 9.48 & 65.69 & 10.81 \\
\hline manure & 140 & 6.42 & 1.84 & 36.75 & 9.81 & 71.14 & 12.09 \\
\hline & 180 & 7.03 & 1.93 & 42.10 & 11.30 & 74.09 & 13.64 \\
\hline \multicolumn{2}{|c|}{ LSD $5 \%$} & 1.99 & 0.39 & 15.41 & 4.90 & 64.12 & 10.69 \\
\hline
\end{tabular}

2- Leaf pigments

\section{a- Main effect of fertilizer type}

Obtained data in Table (6) reveal that conventional fertilization gave significantly the highest values of chlorophyll a, chlorophyll b, total chlorophyll and carotenoids contents. The second fertilizer recorded positive increase in the previous characters was poultry manure followed by cattle manure then sheep manure in both seasons.

Table (6): Main effect of fertilizer type and $\mathrm{N}$ level on broccoli leaf pigments (mg/100mg dry matter) during 2004-2005 and 2005-2006 seasons.

\begin{tabular}{|c|c|c|c|c|c|c|c|c|}
\hline \multirow{2}{*}{$\begin{array}{l}\text { Treatment } \\
\text { Fertilizer }\end{array}$} & $\begin{array}{c}\text { Chl. } \\
\text { a }\end{array}$ & $\begin{array}{c}\text { Chl. } \\
\text { b }\end{array}$ & $\begin{array}{l}\text { Total } \\
\text { Chl. }\end{array}$ & $\begin{array}{c}\text { Carote- } \\
\text { noids }\end{array}$ & $\begin{array}{c}\text { Chl. } \\
\text { a }\end{array}$ & $\begin{array}{c}\text { Chl. } \\
\text { b }\end{array}$ & $\begin{array}{l}\text { Total } \\
\text { Chl. }\end{array}$ & $\begin{array}{c}\text { Carote- } \\
\text { noids }\end{array}$ \\
\hline & \multicolumn{4}{|c|}{ 2004-2005 } & \multicolumn{4}{|c|}{ 2005-2006 } \\
\hline $\begin{array}{l}\text { Fertilizer } \\
\text { Mineral } \\
\text { NPK + cattle manure }\end{array}$ & 2.71 & 1.35 & 4.07 & 0.616 & 2.75 & 1.45 & 4.43 & 0.64 \\
\hline $\begin{array}{l}\text { Poultry manure } \\
\text { Cattle Manure } \\
\text { Sheep manure } \\
\text { LSD 5\% }\end{array}$ & $\begin{array}{l}1.83 \\
1.63 \\
1.5 \\
0.07\end{array}$ & $\begin{array}{l}1.08 \\
0.83 \\
0.67 \\
0.08\end{array}$ & $\begin{array}{l}2.92 \\
2.46 \\
2.28 \\
0.39\end{array}$ & $\begin{array}{c}0.493 \\
0.43 \\
0.3 \\
0.07\end{array}$ & $\begin{array}{l}2.29 \\
1.68 \\
1.59 \\
0.53\end{array}$ & $\begin{array}{l}1.15 \\
0.89 \\
0.71 \\
0.079\end{array}$ & $\begin{array}{l}3.44 \\
2.63 \\
2.57 \\
0.22\end{array}$ & $\begin{array}{l}0.52 \\
0.43 \\
0.31 \\
0.08\end{array}$ \\
\hline $\begin{array}{c}\text { N level (kg/Fed) } \\
100 \\
140 \\
180 \\
\end{array}$ & $\begin{array}{l}1.92 \\
2.03 \\
2.30 \\
\end{array}$ & $\begin{array}{l}0.82 \\
1.01 \\
1.13 \\
\end{array}$ & $\begin{array}{l}2.24 \\
3.12 \\
3.43 \\
\end{array}$ & $\begin{array}{l}0.37 \\
0.48 \\
0.51 \\
\end{array}$ & $\begin{array}{l}1.57 \\
2.19 \\
2.47\end{array}$ & $\begin{array}{l}0.87 \\
1.08 \\
1.22 \\
\end{array}$ & $\begin{array}{l}2.85 \\
3.27 \\
3.66 \\
\end{array}$ & $\begin{array}{l}0.39 \\
0.50 \\
0.54 \\
\end{array}$ \\
\hline LSD 5\% & 0.09 & 0.09 & 0.30 & 0.09 & 0.45 & 0.09 & 0.29 & 0.09 \\
\hline
\end{tabular}

b- Main effect of $\mathbf{N}$ level

Data recorded in Table (6) show that chlorophyll a, chlorophyll b, total chlorophyll and carotenoids contents of leaves were significantly increased with increasing the level of nitrogen from 100 to $180 \mathrm{~kg} /$ Feddan in both growing seasons. 
Hossein, H. A.

The obtained results are in harmony with those of Arjona and Greig (1984) on broccoli, Tarata (1995) on cauliflower and Ampressh-Sharma and Atul-Chandral (2006) on cauliflower.

The favourable effect of nitrogen on leaf pigments formation may be due to nitrogen element being necessary for formation of lipids and chloroplast constituents such as chlorophyll and carotene (Schulze, 1957).

c- Effect of interaction between fertilizer type and $\mathbf{N}$ level

Data in Table (7) show that traditional fertilization containing $180 \mathrm{~kg}$ $\mathrm{N} /$ Feddan gave significantly the highest leaf contents of chlorophyll a, chlorophyll b, total chlorophyll and carotenoids in both seasons.

Table (7): Effect of interaction between fertilizer type and $\mathrm{N}$ level on broccoli leaf pigments $(\mathrm{mg} / 100 \mathrm{mg}$ dry matter) during 20042005 and 2005-2006 seasons.

\begin{tabular}{|c|c|c|c|c|c|c|c|c|c|}
\hline \multirow{2}{*}{\multicolumn{2}{|c|}{ Treatment }} & $\begin{array}{c}\text { Chl. } \\
\text { a }\end{array}$ & $\begin{array}{c}\text { Chl. } \\
\text { b }\end{array}$ & $\begin{array}{l}\text { Total } \\
\text { Chl. }\end{array}$ & $\begin{array}{c}\text { Carote- } \\
\text { noids }\end{array}$ & $\begin{array}{c}\text { Chl. } \\
\text { a }\end{array}$ & $\begin{array}{c}\text { Chl. } \\
\text { b }\end{array}$ & $\begin{array}{l}\text { Total } \\
\text { Chl. }\end{array}$ & $\begin{array}{c}\text { Carote- } \\
\text { noids }\end{array}$ \\
\hline & & \multicolumn{4}{|c|}{ 2004-2005 } & \multicolumn{4}{|c|}{ 2005-2006 } \\
\hline Mineral & 100 & 2.31 & 1.14 & 3.45 & 0.49 & 2.56 & 1.23 & 3.79 & 0.51 \\
\hline \multirow{2}{*}{$\begin{array}{l}\text { NPK + } \\
\text { cattle } \\
\text { manure }\end{array}$} & 140 & 2.75 & 1.39 & 4.14 & 0.67 & 3.02 & 1.49 & 4.51 & 0.69 \\
\hline & 180 & 3.09 & 1.52 & 4.61 & 0.69 & 3.35 & 1.64 & 4.99 & 0.73 \\
\hline \multirow{3}{*}{$\begin{array}{l}\text { Poultry } \\
\text { Manure }\end{array}$} & 100 & 0.94 & 0.96 & 1.91 & 0.40 & 1.93 & 1.01 & 2.94 & 0.42 \\
\hline & 140 & 2.15 & 1.08 & 3.23 & 0.52 & 2.30 & 1.14 & 3.44 & 0.55 \\
\hline & 180 & 2.42 & 1.22 & 3.64 & 0.56 & 2.64 & 1.31 & 3.95 & 0.59 \\
\hline Cattle & 100 & 1.31 & 0.67 & 1.98 & 0.39 & 1.41 & 0.71 & 2.12 & 0.40 \\
\hline \multirow{2}{*}{ manure } & 140 & 1.69 & 0.88 & 2.57 & 0.41 & 1.81 & 0.94 & 2.75 & 0.43 \\
\hline & 180 & 1.89 & 0.94 & 2.83 & 0.44 & 2.01 & 1.00 & 3.01 & 0.47 \\
\hline Sheep & 100 & 1.15 & 0.49 & 1.64 & 0.22 & 1.23 & 0.51 & 2.52 & 0.23 \\
\hline \multirow[t]{2}{*}{ manure } & 140 & 1.54 & 0.68 & 2.22 & 0.32 & 1.64 & 0.73 & 2.37 & 0.34 \\
\hline & 180 & 1.81 & 0.83 & 2.64 & 0.36 & 1.91 & 0.88 & 2.79 & 0.37 \\
\hline LSD 5\% & & 0.19 & 0.20 & 0.60 & 0.19 & 0.90 & 0.19 & 0.59 & 0.19 \\
\hline
\end{tabular}

3- Leaves mineral composition

\section{a- Main effect of fertilizer type}

In general, conventional fertilization recorded significantly higher N, P and $\mathrm{K}$ contents of leaves than organic manures. Poultry manure gave significantly the highest values of $\mathrm{N}, \mathrm{P}$ and $\mathrm{K}$ contents. The second organic manure with positive increase in the previous parameters was cattle manure followed by sheep manure in both seasons (Table 8). Similar results were obtained on onion by Lallangoud et al. (1999) and Mahmoud (2006), where they reported that plant from plots treated with the recommended rates of $\mathrm{N}$, $\mathrm{P}$ and $\mathrm{K}$ exhibited higher uptake of $\mathrm{N}, \mathrm{P}$ and $\mathrm{K}$ than those grown under organic production. Furthermore, El-Mansi et al. (2004) indicated that application of chicken manure increased pea leaves $\mathrm{N}, \mathrm{P}$ and $\mathrm{K}$ contents compared with application of cattle manure.

\section{b- Main effect of $\mathbf{N}$ level}

Data in Table (8) reveal that N, P and $\mathrm{K}$ contents were significantly increased with increasing $\mathrm{N}$ level from 100 to $180 \mathrm{~kg} /$ Feddan except $\mathrm{P}$ content in both seasons and $\mathrm{K}$ content in the second season.

\section{c- Effect of interaction between fertilizer type and $\mathrm{N}$ level}


Traditional fertilization containing $180 \mathrm{~kg} \mathrm{~N} /$ Feddan gave significantly the highest N, P and K contents of leaves in both seasons (Table 9).

Table (8): Main effect of fertilizer type and $\mathrm{N}$ level on chemical composition of broccoli leaves during 2004-2005 and 20052006 seasons.

\begin{tabular}{|c|c|c|c|c|c|c|}
\hline Character & $\mathbf{N}(\%)$ & $\begin{array}{c}P(\%) \\
2044-2005 \\
\end{array}$ & K (\%) & $\mathbf{N}(\%)$ & $\begin{array}{c}P(\%) \\
2005-2006 \\
\end{array}$ & K (\%) \\
\hline $\begin{array}{l}\text { Fertilizer } \\
\text { Mineral } \\
\text { NPK + cattle manure }\end{array}$ & 2.61 & 0.508 & 1.791 & 2.75 & 0.544 & 1.81 \\
\hline Poultry manure & 2.08 & 0.367 & 1.613 & 2.21 & 0.39 & 1.71 \\
\hline Cattle Manure & 1.94 & 0.229 & 1.57 & 1.84 & 0.307 & 1.65 \\
\hline Sheep manure & 1.20 & 0.267 & 1.34 & 1.27 & 0.262 & 1.44 \\
\hline $\begin{array}{c}\text { LSD 5\% } \\
\text { N level (kg/Fed.) }\end{array}$ & 0.154 & 0.076 & 0.08 & 0.15 & 0.078 & 0.15 \\
\hline 100 & 1.76 & 0.319 & 1.46 & 1.84 & 0.342 & 1.56 \\
\hline 140 & 2.03 & 0.353 & 1.62 & 2.01 & 0.362 & 1.67 \\
\hline 180 & 2.89 & 0.396 & 1.66 & 2.21 & 0.424 & 1.73 \\
\hline LSD 5\% & 0.19 & NS & 0.06 & 0.19 & NS & NS \\
\hline
\end{tabular}

Table (9): Effect of interaction between fertilizer type and $\mathbf{N}$ level on chemical composition of broccoli leaves during 2004-2005 and 2005-2006 seasons.

\begin{tabular}{|c|c|c|c|c|c|c|c|}
\hline \multicolumn{2}{|r|}{ acter } & $\mathbf{N}(\%)$ & $\mathbf{P}(\%)$ & K (\%) & $\mathbf{N}(\%)$ & P (\%) & K (\%) \\
\hline \multicolumn{2}{|l|}{ Treatment } & \multicolumn{3}{|c|}{ 2044-2005 } & \multicolumn{3}{|c|}{ 2005-2006 } \\
\hline Mineral & 100 & 2.41 & $20+8000$ & 1.78 & 2.53 & 0.484 & 1.71 \\
\hline \multirow{2}{*}{$\begin{array}{c}\text { NPK + cattle } \\
\text { manure }\end{array}$} & 140 & 2.68 & 0.512 & 1.90 & 2.85 & 0.544 & 1.84 \\
\hline & 180 & 2.75 & 0.569 & 1.96 & 2.85 & 0.604 & 1.88 \\
\hline \multirow{3}{*}{$\begin{array}{l}\text { Poultry } \\
\text { manure }\end{array}$} & 100 & 1.81 & 0.309 & 1.66 & 1.89 & 0.339 & 1.61 \\
\hline & 140 & 2.18 & 0.357 & 1.75 & 2.34 & 0.376 & 1.72 \\
\hline & 180 & 2.25 & 0.438 & 1.83 & 2.39 & 0.455 & 1.79 \\
\hline \multirow{3}{*}{$\begin{array}{l}\text { Cattle } \\
\text { Manure }\end{array}$} & 100 & 1.72 & 0.289 & 1.63 & 1.78 & 0.289 & 1.61 \\
\hline & 140 & 2.01 & 0.274 & 1.70 & 1.55 & 0.292 & 1.65 \\
\hline & 180 & 2.09 & 0.299 & 1.79 & 2.19 & 0.327 & 1.69 \\
\hline \multirow{3}{*}{$\begin{array}{l}\text { Sheep } \\
\text { manure }\end{array}$} & 100 & 1.08 & 0.254 & 1.33 & 1.14 & 0.274 & 1.31 \\
\hline & 140 & 1.23 & 0.268 & 1.51 & 1.31 & 0.234 & 1.48 \\
\hline & 180 & 1.30 & 0.281 & 1.58 & 1.36 & 0.296 & 1.54 \\
\hline \multicolumn{2}{|c|}{ LSD 5\% } & 0.39 & 0.197 & 0.11 & 0.40 & 0.197 & 0.40 \\
\hline
\end{tabular}

4- Yield and its components

\section{a- Main effect of fertilizer type}

Data in table (10 and 12) show that conventional fertilization gave the highest values of main spear yield, average side spear diameter, number of side spear per plant, side spear yield and total yield. The second treatment with positive increase in the previous parameters was poultry manure, followed by cattle manure then sheep manure, in both seasons. Application of $180 \mathrm{~kg} \mathrm{~N} /$ Fed increased total yield by $17.07 \%$ and $65.53 \%$, while $140 \mathrm{~kg}$ $\mathrm{N} /$ Fed increased total yield by $14.38 \%$ and $58.77 \%$ over $100 \mathrm{~kg} \mathrm{~N} /$ Fed in the 
Hossein, H. A.

first and second seasons, respectively. Concerning total yield, similar results were obtained by Mallanagouda et al. (1995) on garlic, where they reported that plants from plots treated with mineral $\mathrm{N}, \mathrm{P}$ and $\mathrm{K}$ fertilizers exhibited higher total yield than farmyard manure. Furthermore, the obtained results agree with those of Arenfalk and Hogelskjoor (1995) on leek, Mohamed and Gamie (1999) and Hossein (2000) and Mahmoud (2006) on onion. Respecting the effect of different organic manures on total yield, the obtained results agree with those of Abd El-Mouty et al. (2000) on squash where they reported that poultry manure gave higher yield than cattle manure.

The observed enhancement on broccoli yield with application of conventional fertilization as compared with organic manure may be attributed to the increase in vegetative growth (Tables 2, 4), in leaf pigments (Table 6) and in leaves chemical composition (Table 8).

Table (10): Main effect of fertilizer type and $N$ level on broccoli yield and its components during 2004-2005 season.

\begin{tabular}{|c|c|c|c|c|c|c|c|c|}
\hline Character & $\begin{array}{c}\text { Main } \\
\text { spear } \\
\text { diameter } \\
(\mathrm{cm})\end{array}$ & $\begin{array}{l}\text { Main } \\
\text { spear } \\
\text { rweight } \\
\text { (g) }\end{array}$ & $\begin{array}{l}\text { Main } \\
\text { spears } \\
\text { yield } \\
\text { (Ton/ } \\
\text { Fed) }\end{array}$ & $\begin{array}{l}\text { Average } \\
\text { side } \\
\text { spear } \\
\text { diameter } \\
\text { (cm) }\end{array}$ & $\begin{array}{l}\text { No. of } \\
\text { side } \\
\text { spear } \\
\text { per plant }\end{array}$ & $\begin{array}{l}\text { Side } \\
\text { spears } \\
\text { yield } \\
\text { per } \\
\text { plant } \\
\text { (g) }\end{array}$ & $\begin{array}{c}\text { Side } \\
\text { spears } \\
\text { yield } \\
\text { (Ton/ } \\
\text { Fed) }\end{array}$ & $\begin{array}{l}\text { Total } \\
\text { yield } \\
\text { (Ton/ } \\
\text { Fed) }\end{array}$ \\
\hline \multicolumn{9}{|l|}{ Fertilizer } \\
\hline $\begin{array}{l}\text { Mineral NPK + cattle } \\
\text { manure }\end{array}$ & 11.70 & 468.24 & 4.265 & 5.132 & 6.69 & 130.53 & 1.083 & 5.352 \\
\hline Poultry manure & 11.30 & 440.54 & 4.057 & 4.746 & 5.08 & 91.28 & 0.861 & 4.907 \\
\hline Cattle Manure & 10.34 & 289.04 & 2.801 & 3.864 & 4.36 & 77.29 & 0.679 & 3.183 \\
\hline Sheep manure & 9.08 & 240.23 & 2.225 & 2.554 & 3.12 & 45.99 & 0.411 & 2.635 \\
\hline $\begin{array}{l}\text { LSD } 5 \% \\
\text { N level } \\
\text { (kg/Fed.) }\end{array}$ & 0.77 & 41.63 & 0.654 & 0.335 & 0.32 & 7.39 & 0.08 & 0.38 \\
\hline 100 & 9.58 & 334.95 & 2.975 & 3.712 & 4.33 & 76.06 & 0.667 & 3.638 \\
\hline 140 & 10.86 & 365.04 & 3.420 & 4.132 & 4.87 & 88.34 & 0.791 & 4.161 \\
\hline 180 & 11.38 & 378.55 & 3.616 & 4.378 & 5.24 & 100.43 & 0.819 & 4.259 \\
\hline LSD 5\% & 0.99 & NS & 0.413 & 0.368 & 0.43 & 12.33 & 0.09 & 0.499 \\
\hline
\end{tabular}

\section{b- Main effect of $\mathrm{N}$ level}

Data in Tables (10 and 12) reveal that the parameters of yield and its components were significantly increased with increasing $\mathrm{N}$ level from 100$180 \mathrm{~kg} /$ Feddan, except main spear weight in both seasons and main spear yield and side spears weight per plant in the second season. Also, data showed that respecting total yield, the obtained results are in accordance with those of Arjana and Greig (1984) on broccoli, Yadav and Paliwal (1990) on cauliflower, Zebarth et al. (1995) on broccoli, Balyan et al. (1994) on cauliflower, Abdul Baki et al. (1997) on broccoli, Kaniszewsk and Willigen (1998) on cauliflower, Fueraarts and Willigen (1999) on broccoli, Babik and El-Kner (2002) on broccoli, Jana and Mukheredhyay (2002) on cauliflower, Brahama et al. (2002) on broccoli and Kuldeep-Singh et al. (2004) on cauliflower. Increasing total yield with increasing $\mathrm{N}$ level may be explained through the significant role of $\mathrm{N}$ element in enhancing plant growth (Tables 2 and 4). In addition, it has an indispensable role in photosynthetic pigments 
formation (Table 6). This in turn, stimulate the net assimilation rate and increased total yield.

c- Effect of interaction between fertilize type and $\mathrm{N}$ level

Obtained data in Tables (11 and 13) show that conventional fertilization containing $140 \mathrm{~kg} \mathrm{~N} /$ Feddan gave significantly the highest values of main spear diameter, main spear weight, main spears yield, average side spear diameter, side spears yield per plant, weight of side spears per plant, side spear yield and total yield in both seasons.

Table (11): Effect of interaction between fertilizer type and $N$ level on broccoli yield and its components during 2004-2005 season.

\begin{tabular}{|c|c|c|c|c|c|c|c|c|c|}
\hline \multicolumn{2}{|c|}{ Character } & $\begin{array}{l}\text { Main } \\
\text { spear } \\
\text { diameter } \\
(\mathrm{cm})\end{array}$ & $\begin{array}{l}\text { Main } \\
\text { spear } \\
\text { weight } \\
\text { (g) }\end{array}$ & $\begin{array}{l}\text { Main } \\
\text { spears } \\
\text { yield } \\
\text { (Ton/ } \\
\text { Fed) }\end{array}$ & $\begin{array}{l}\text { Average } \\
\text { side } \\
\text { spear } \\
\text { diameter } \\
\text { (cm) }\end{array}$ & $\begin{array}{l}\text { No. of } \\
\text { side } \\
\text { spear } \\
\text { Per } \\
\text { plant }\end{array}$ & $\begin{array}{c}\text { Side } \\
\text { spears } \\
\text { yield } \\
\text { Per plant } \\
\text { (g) }\end{array}$ & $\begin{array}{l}\text { Side } \\
\text { spears } \\
\text { yield } \\
\text { (Ton/ } \\
\text { Fed) }\end{array}$ & $\begin{array}{l}\text { Total } \\
\text { yield } \\
\text { (Ton/ } \\
\text { Fed) }\end{array}$ \\
\hline \multirow{3}{*}{$\begin{array}{c}\text { Mineral } \\
\text { NPK + } \\
\text { cattle } \\
\text { manure }\end{array}$} & 100 & 11.09 & 459.80 & 4.418 & 4.720 & 5.88 & 112.85 & 0.984 & 5.702 \\
\hline & 140 & & & 4.598 & & & 185.71 & 1.236 & \\
\hline & 180 & 9.703 & 428.46 & 3.791 & 5.317 & 6.78 & 117.21 & 1.030 & 4.821 \\
\hline \multirow{3}{*}{$\begin{array}{l}\text { Poultry } \\
\text { manure }\end{array}$} & 100 & 10.07 & 418.90 & 3.478 & 3.808 & 4.81 & 86.36 & 0.751 & 4.229 \\
\hline & 140 & 11.19 & & 4.272 & 4.594 & 4.59 & 94.82 & 0.840 & 5.112 \\
\hline & 180 & 12. & 459.7 & 4.388 & 5.170 & 5.84 & 92.66 & 0.991 & 5.379 \\
\hline \multirow{3}{*}{$\begin{array}{l}\text { Cattle } \\
\text { Manure }\end{array}$} & 100 & 9.1 & 258.7 & 2.127 & & 3.98 & 68. & 0.596 & 2.723 \\
\hline & 140 & 10.5 & 269.01 & 2.374 & 4.005 & 4.38 & 74.92 & 0.672 & 3.046 \\
\hline & 180 & 11.29 & 339.5 & 3.009 & 4.148 & 4.72 & 88.64 & 0.770 & 3.779 \\
\hline \multirow{3}{*}{$\begin{array}{l}\text { Sheep } \\
\text { manure }\end{array}$} & 100 & 7.98 & 212.35 & 1.864 & 2.215 & 2.66 & 36.71 & 0.335 & 2.199 \\
\hline & 140 & & & 2.236 & & 3.0 & 46. & 0.414 & 2.650 \\
\hline & 180 & 9.83 & & 2.574 & 2.878 & 3.62 & 54.83 & 0.483 & 3.057 \\
\hline & 1.99 & 102.16 & 0.827 & 0.737 & 0.85 & 24.66 & 0.193 & 0.99 \\
\hline
\end{tabular}

Table (12): Main effect of fertilizer type and $N$ level on broccoli yield and its components during 2005-2006 season.

\begin{tabular}{l|cccccccc}
\hline & $\begin{array}{c}\text { Main spear } \\
\text { diameter } \\
\text { (cm) }\end{array}$ & $\begin{array}{c}\text { Main } \\
\text { spear } \\
\text { weight } \\
\text { (g) }\end{array}$ & $\begin{array}{c}\text { Main } \\
\text { spears } \\
\text { yield } \\
\text { (Ton/ } \\
\text { Fed) }\end{array}$ & $\begin{array}{c}\text { Average } \\
\text { side } \\
\text { spear } \\
\text { diameter } \\
\text { (cm) }\end{array}$ & $\begin{array}{c}\text { No. of } \\
\text { side } \\
\text { spear } \\
\text { per plant }\end{array}$ & $\begin{array}{c}\text { Side } \\
\text { spears } \\
\text { yield } \\
\text { per } \\
\text { plant } \\
\text { (g) }\end{array}$ & $\begin{array}{c}\text { Side } \\
\text { spears } \\
\text { yield } \\
\text { Treatment }\end{array}$ & $\begin{array}{c}\text { Total } \\
\text { yield } \\
\text { (Ton) }\end{array}$ \\
Fed)
\end{tabular}

Table (13): Effect of interaction between fertilizer type and $\mathrm{N}$ level on broccoli yield and its components during 2005-2006 season. 
Hossein, H. A.

\begin{tabular}{|c|c|c|c|c|c|c|c|c|}
\hline Treatment & $\begin{array}{c}\text { Main } \\
\text { spear } \\
\text { diameter } \\
(\mathbf{c m})\end{array}$ & $\begin{array}{l}\text { Main } \\
\text { spear } \\
\text { weight } \\
\text { (g) }\end{array}$ & $\begin{array}{l}\text { Main } \\
\text { spears } \\
\text { yield } \\
\text { (Ton/ } \\
\text { Fed) }\end{array}$ & $\begin{array}{l}\text { Average } \\
\text { side } \\
\text { spear } \\
\text { diameter } \\
\text { (cm) }\end{array}$ & $\begin{array}{l}\text { No. of } \\
\text { side } \\
\text { spear } \\
\text { per } \\
\text { plant }\end{array}$ & $\begin{array}{l}\text { Side } \\
\text { spears } \\
\text { yield } \\
\text { per } \\
\text { plant } \\
\text { (g) }\end{array}$ & $\begin{array}{c}\text { Side } \\
\text { spears } \\
\text { yield } \\
\text { (Ton/ } \\
\text { Fed) }\end{array}$ & $\begin{array}{l}\text { Total } \\
\text { yield } \\
\text { (Ton/ } \\
\text { Fed) }\end{array}$ \\
\hline Mineral 100 & 1 & 41805 & 3.714 & 111 & 515 & 108.57 & 0.950 & 4.664 \\
\hline NPK + 140 & & & & & & & & \\
\hline $\begin{array}{c}\text { cattle } \\
\text { manure }\end{array}$ & 9.73 & 386.12 & 3.429 & 4.63 & 5.87 & 111.96 & 0.995 & 4.424 \\
\hline Poultry 100 & 9.15 & 355.77 & & & & 81.92 & 0.720 & 3.870 \\
\hline $\begin{array}{ll} & 140 \\
\text { manure } & 180\end{array}$ & $\begin{array}{c}9.26 \\
11.48\end{array}$ & $\begin{array}{l}402.75 \\
415.42\end{array}$ & & & & $\begin{array}{l}90.22 \\
87.25\end{array}$ & & $\begin{array}{l}4.378 \\
4.558\end{array}$ \\
\hline 100 & 8.33 & 217.50 & 1.930 & 3.00 & 3.52 & 64.87 & 0.576 & 2.506 \\
\hline 140 & 8.71 & 225.63 & 1.999 & & 3.89 & 71.83 & & 2.63 \\
\hline 180 & 10.25 & & & & & 76.81 & & 3.233 \\
\hline 100 & 7.25 & 178.11 & 1.582 & 2.11 & 2.30 & 35.65 & 0.317 & 1.897 \\
\hline 140 & 8.58 & & 1.874 & 2.4 & & & & 8.264 \\
\hline 180 & & & & & & 52.02 & & 2.612 \\
\hline LSD 5\% & 2.25 & 99.34 & 0.599 & 0.599 & 0.85 & 21.99 & 0.199 & 0.712 \\
\hline
\end{tabular}

\section{5- Spear pigments}

\section{a- Main effect of fertilizer type}

Data in Table (14) illustrate that conventional fertilizers gave significantly the highest chlorophyll a, chlorophyll b, total chlorophyll and carotenoids contents of spear followed by poultry manure then cattle manure, while sheep manure recorded the lowest values of the previous parameters in both seasons.

Table (14): Main effect of fertilizer type and $\mathrm{N}$ level on spear pigments (mg/g fresh matter) of broccoli during 2004-2005 and 20052006 seasons.

\begin{tabular}{|c|c|c|c|c|c|c|c|c|}
\hline Che & $\begin{array}{c}\text { Chl. } \\
\text { a }\end{array}$ & $\begin{array}{c}\text { Chl. } \\
\text { b }\end{array}$ & $\begin{array}{l}\text { Total } \\
\text { Chl. }\end{array}$ & $\begin{array}{l}\text { Carot- } \\
\text { enoids }\end{array}$ & $\begin{array}{c}\text { Chl. } \\
\text { a }\end{array}$ & $\begin{array}{c}\text { Chl. } \\
\text { b }\end{array}$ & $\begin{array}{l}\text { Total } \\
\text { Chl. }\end{array}$ & $\begin{array}{l}\text { Carot- } \\
\text { enoids }\end{array}$ \\
\hline Treatment & & 2004 & -2005 & & & 200 & $5-2006$ & \\
\hline Fertilizer & & & & & & & & \\
\hline $\begin{array}{l}\text { Mineral NPK } \\
\text { cattle manure }\end{array}$ & +0.494 & 0.313 & 0.806 & 0.293 & 0.545 & 0.339 & 0.885 & 0.319 \\
\hline Poultry manure & 0.466 & 0.274 & 0.738 & 0.233 & 0.512 & 0.300 & 0.812 & 0.250 \\
\hline Cattle Manure & 0.414 & 0.261 & 0.694 & 0.210 & 0.461 & 0.285 & 0.246 & 0.222 \\
\hline Sheep manure & 0.387 & 0.234 & 0.615 & 0.159 & 0.415 & 0.257 & 0.678 & 0.174 \\
\hline $\begin{array}{c}\text { LSD \% } \\
\mathrm{N} \text { level }(\mathrm{kg} / \text { Fed) }\end{array}$ & 0.063 & 0.022 & 0.069 & 0.027 & 0.052 & 0.019 & 0.073 & 0.019 \\
\hline 100 & 0.384 & 0.211 & 0.594 & 0.184 & 0.422 & 0.229 & 0.651 & 0.198 \\
\hline 140 & 0.449 & 0.273 & 0.718 & 0.2 & 0.495 & 0.299 & 0.795 & 0.247 \\
\hline 180 & 0.488 & 0.328 & 0.827 & 0.2 & 0.533 & 0.358 & 0.896 & 0.279 \\
\hline LSD 5\% & 0.488 & 0.030 & 0.088 & 0.099 & 0.067 & 0.026 & 0.094 & 0.026 \\
\hline
\end{tabular}

b- Main effect of $\mathbf{N}$ level 
Data in Table (14) show that chlorophyll a, chlorophyll b, total chlorophyll and carotenoids contents were significantly increased with increasing $\mathrm{N}$ level in the fertilizer from 100 to $180 \mathrm{~kg} /$ Feddan.

\section{c- Effect of interaction between fertilizer type and $\mathrm{N}$ level}

Data in Table (15) show that the conventional fertilization containing $180 \mathrm{~kg} /$ Feddan gave significantly the highest values of the previous characters in both seasons.

In conclusion, the present investigation demonstrated that conventional fertilization containing $140 \mathrm{~kg} \mathrm{~N} /$ Feddan gave the highest growth and yield and that poultry manure containing $180 \mathrm{~kg} \mathrm{~N} /$ Feddan gave higher growth and yield than the other organic manures.

Table (15): Effect of interaction between fertilizer type and $N$ level on spear pigments $(\mathrm{mg} / \mathrm{g}$ fresh matter) of broccoli during 20042005 and 2005-2006 seasons.

\begin{tabular}{|c|c|c|c|c|c|c|c|c|c|}
\hline \multirow{2}{*}{\multicolumn{2}{|c|}{$\begin{array}{l}\text { Character } \\
\text { Treatment }\end{array}$}} & $\begin{array}{c}\text { Chl. } \\
\text { a }\end{array}$ & $\begin{array}{c}\text { Chl. } \\
\text { b }\end{array}$ & $\begin{array}{l}\text { Total } \\
\text { Chl. }\end{array}$ & $\begin{array}{l}\text { Carot- } \\
\text { enoids }\end{array}$ & $\begin{array}{c}\text { Chl. } \\
\text { a }\end{array}$ & $\begin{array}{c}\text { Chl. } \\
\text { b }\end{array}$ & $\begin{array}{l}\text { Total } \\
\text { Chl. }\end{array}$ & $\begin{array}{l}\text { Carot- } \\
\text { enoids }\end{array}$ \\
\hline & & \multicolumn{4}{|c|}{ 2004-2005 } & \multicolumn{4}{|c|}{ 2005-2006 } \\
\hline \multicolumn{2}{|c|}{ Mineral 100} & 0.450 & 0.251 & 0.701 & 0.241 & & 0.271 & & \\
\hline \multirow{4}{*}{$\begin{array}{l}\text { NPK + } \\
\text { cattle } \\
\text { manure } \\
\text { Poultry } \\
\text { Manure }\end{array}$} & 140 & & & 0.806 & & & 0 & & \\
\hline & 180 & 0.537 & 0.375 & 0.912 & 0.344 & 0.587 & 0.406 & 0.993 & 0.378 \\
\hline & 100 & 0.424 & 0.224 & 0.648 & 0.201 & 0.461 & 0.245 & 0.7 & 0. \\
\hline & $\begin{array}{l}140 \\
180\end{array}$ & $\begin{array}{l}0.46 \\
0.50\end{array}$ & & & & & & & \\
\hline & 100 & 0.34 & 0.205 & & & & & & \\
\hline \multirow[t]{2}{*}{ manure } & 140 & 0.427 & 0.2 & 0.65 & & 0.4 & 0.28 & & 0.2 \\
\hline & 180 & $0.4 c$ & & & & & & & \\
\hline neep & 100 & & & & & & & & \\
\hline \multirow[t]{2}{*}{ manure } & 140 & & & & & & & & \\
\hline & 180 & 0.43 & & & & 0.47 & 0.322 & & \\
\hline \multicolumn{2}{|l|}{ LSD 5\% } & 0.088 & 0.059 & 0.173 & 0.139 & 0.134 & 0.052 & 0.189 & 0.052 \\
\hline
\end{tabular}

\section{REFERENCES}

Abdel El-Mouty, M.M.A; A.H. Ali and Fatma, A. Rizk (2000). The organic production of squash (Cucurbita pepo L.) as effected by different sources of organic fertilizers, Egypt, J. Appl. Sci.. 15 (8): 159-177.

Abdul Baki, A.A.; R.D. Morse; J.R. Teasdale and T.E. Devine (1997). Nitrogen requirements of broccoli in cover crop mulches and clean cultivation. Journal of vegetable crop production. 3 (2): 85-100.

Ali, A.H.; M.M. Abdel-Mouty and A.M. Shaheen (2001). Effect of BioNitrogen, organic and in-organic fertilizer on the productivity of garlic (Allium sativum L.) plants.

Amreesh-Sharma and Atul-Chandra (2006). Effect of plant density and nitrogen levels on physico-chemical parameters of cauliflower. Haryana Journal of Horticultural Sciences. 33 (1/2): 148-149.

Arjona, D.H. and J. Greig (1984). Study of the effect of four planting distances and four levels of nitrogen fertilization on yields, chlorophyll 
Hossein, H. A.

content and nitrate reductase activity in broccoli. Agronomia. Colombiana. 2 (1/2): 71-81.

Babik, I. And K. Elkner (2002). The effect of nitrogen fertilization and irrigation on yield and quality of broccoli. Acta-Horti. (571): 33-43.

Balyan, D.S.; Joginder-Singh and V.K. Srivastara (1994). Nitrogen and zinc interactions in Cauliflower. Crop Research Hisar. 8(3): 537-542.

Borin, M.C.; Giupponi and Osele (1987). The effect of organic and mineral fertilizer and soil type on potato tuber formation. Information Agrario, 43: 82-87.

Brahma, S.D.B. Phookan and B.P. Gautam (2002). Effect of nitrogen, phosphorus and potassium on growth and yield of broccoli (Brassica Oleracea L. Var. italica) cv. Pusa broccoli K Ts.1. Journal of the Agricultural Science Society of North East India. 15(1): 104-106.

Bremner, J.M. and C.S. Mulvaney (1992). Total nitrogen in page (A.L. R.H. Miller and D.R. Keeney (Eds). Methods of Soils Analysis, Part 2 Amer. Soc. Agron. Madson WI.W.S.A. pp. 595-624).

Browaldh, M. (1992). Influence of organic and inorganic fertilizer on common bean, (Phaeolus vulgaris L.) grown in a-p-fixing moilic adosol. Biol. Agric. And hort., 9:87.

El Mansi, A.A.; H.M. Arisha and A.I. El-Kassas (2004). Effect of organic manure sources on growth, chemical contents and yield of pea plants under sandy soil conditions. Zagazig J. Agric. Res., Vol. 31, No. (5): 2097-2121.

El-Sheikh, T.M. and G.M. Salama (1997). Influence of chicken manure on growth, quality and storageability of tomato. Annals of Agric. Sc. Moshtohor, Vol. 35(4): 2391-2413.

Everaarts, A.P. and P. de-Willigen (1999). The effect of nitrogen and the method of application on yield and quality of broccoli. Netherlands Journal of Agricultural Science. 47 (2): 123-133.

Fisher, A. and C. Richter (1984). Influence of organic and mineral fertilizers on yield and quality of potatoes. The fifth Ifo international Scientific Conference at the University of KA Germany. P.37

Gliessman, S.R.; M.R. Werner; J. Allison and J. Cohran (1996). A comparison of strawberry plant development and yield under organic and conventional management on the central California Coast. Biological Agric. And Hort., 12, pp. 327-338.

Gomez, K.E.A. and A.A. Gomez (1984). Statistical Procedures for the Agricultural Researches, John Wiley and Son, Inc., New York.

Hossein, H.E.A (2002). Effect of some agricultural treatments on productivity and quality of onion grown in Sandy Soils. Ph.D. Thesis, Faculty of Agric., Suez Canal

Horlink, H.A.J.; Y. Inhar; M.J. Bolham (1991). States of compost amended Potting mixes naturaily suppressive to soil born diseases of horticulture crops. Plant diseases, 23: 869-873.

Kaniszewski, S. and J. Rumpel (1998). Effects of irrigation, nitrogen fertilization and soil type on yield and quality of cauliflower. Journal of vegetable Crop production. 4(1): 67-75. 
Mahmoud M.R. (2006). Effect of some organic and inorganic nitrogen fertilization on onion plant, grown on sandy calcareous soil, Assiut $\mathrm{J}$. Agric. Sci., Vol. 37, No.1: 147-159.

Mallanagouda, B.S.G.S.; N.C. Hulamani; B.C. Murthy and B.B. Madalgere (1995). Effect of NPK and FYM on growth parameters on onion, garlic and coriander. Current research Univ., of Agric. Sciences, Bangalore, 24 (11): 212-213.

Marschner, H. (1988). Mineral Nutrition of High plants $2^{\text {nd }}$ ed. Institute of Plant Nutrition Univ. of Hohenheim Germany.

Meissner-Smejkal, G. (2000). Soil microbial activities in organic and conventional vegetable gardening. A sensitive instrument of horticultural research, Acta. Hort., 513: 145-152.

Mohamed, E.L. and A.A. Gamie (1999). Evaluation of some organic fertilizers as substitutions of chemical fertilizers in fertilizing onion. Egypt J. Appl. Sci., 14(7): 664-678.

Olsen, S.R. and L.E. Sommers (1982). Phosphorus. In: Page A.L.R. Miller and D.R. Keeney (Eds) Methods of Soil Analysis, Partz Amer. Soc. Agron Madison WI. USA. Pp. 403-430.

Schulze, W. (1957). Unburden Einfluss der Dungung auf die Bildung der Chloroplasten, Pigment Pflanzenernahr. Dung Bodenkd. 76, 1-19.

Sharma, S.K.; Rajender-Sharma and B.N. Korla (2002). Effect of nitrogen and phosphorus on the growth and seed yield of sprouting broccoli CV. Green Head. Horticultural Journal. 15(2): 87 - 90.

Singh, N.K. and Akhilesh-Singh (2000). Influence of nitrogen and potassium on growth and head yield of broccoli (Btassica Oleracea L. var. italica) under low hills subtropical condition of H.P. Vegetable Science. 27(1): 99-100.

Skoog, F. (1979). Plant growth substances. Proceeding of the $10^{\text {th }}$ international conference on plant growth substances, Madison, Wisconsin. July, 22-26.

Sendecor, G.W. and W.G. Cochran (1980). Statistical methods. 7thed. IOWA State Univ. press, Amer. IOWA. USA.

Jockon, M.I.A (1990). Soil chemical Analysis. Prentice Hall. Englewood cliffs, N.J.

Jana, J.C. and T.P mukhopadhyay (2007). Effect of nitrogen and phosphprus on growth and curd yield of vegetables. Science. 28 (2): 133-136.

Tarata, G; N. Popandren; M. Podoleanm; M. Gavriliuc and J. munteau (1995). Studies on effect of nitrogen Fertilizers on cabbage and cauliflower. Anale institutual de cercetari pentrm legumicultura $\mathrm{Si}$ floriculture, Vidra 13: 472- 484.

Wettestein, D.V. (1957). Chlorophyll. Lethal and under submikrosskopische from wechsel der plastid. Expt cell Res. 12: 427-206.

Yadav, B.R. and K.V. Paliwal (1990). Response of cauliflower to nitrogen and phosphorus fertilization on irrigation with saline waters. VegetableScience 17 (1): 1-6.

Zebrth, B.J.; P.A. Bowen and P.M.A. Toivnen (1995). Influence of nitrogen fertilization on broccoli yield, nitrogen accumulation and apparent 
Hossein, H. A.

fertilizer- nitrogen recovery. Canadian Journal of Plant Science. 75 (3): 717-725.

تأثير معدلات مختلفة من الأسمدة العضوية والتسميد التقليدى على النمو الخضرى التصى

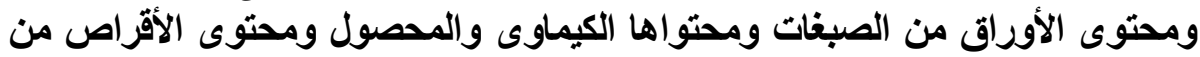
الصبغات في البروكلي

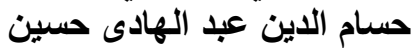
قسم البساتين - كلية الزراعة بالإسماعيلية ـ جامعة قناة السويس

أجريت هذه التجربة على نباتات البروكلى خلال موسمى 2004 - 2005, 2005 -

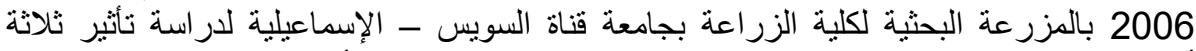

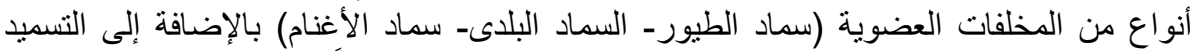

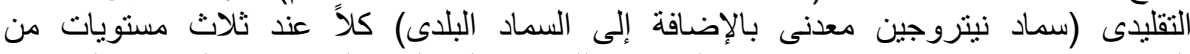

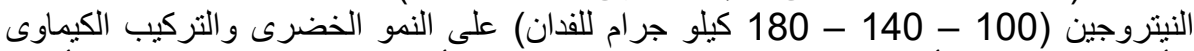

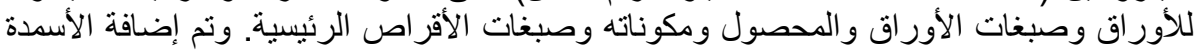

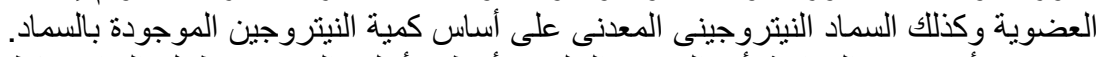

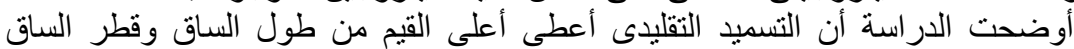

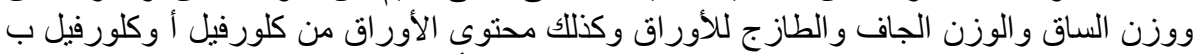

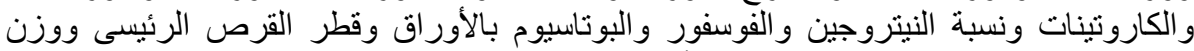

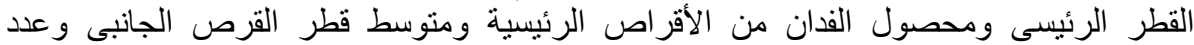

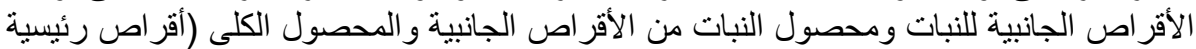

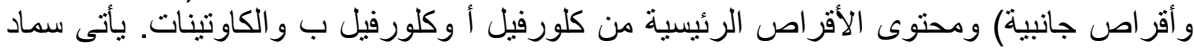

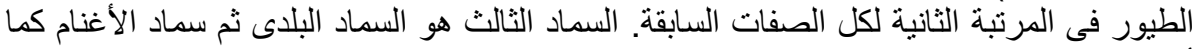

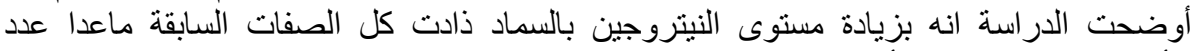

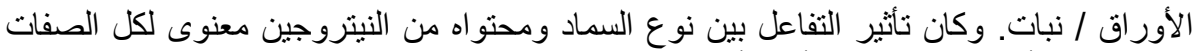

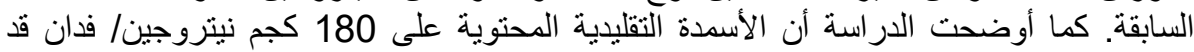

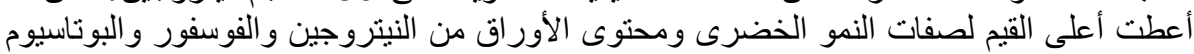

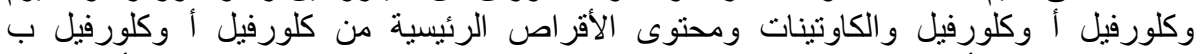

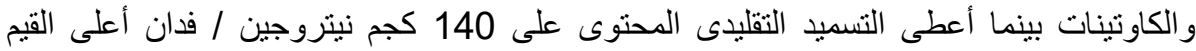

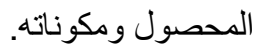


J. Agric. Sci. Mansoura Univ., 33 (3), March, 2008 


\begin{tabular}{|c|c|}
\hline ABSTRACT & الملخص ال المص \\
\hline INTRODUCTION & المقدمة المقة \\
\hline MATERIALS AND METHODS & الأسلوب البحثى \\
\hline RESULTS AND DISCUSSION & النتائج ومناقشتها \\
\hline REFERENCES & المراجـع \\
\hline ABSTRACT & الملخص \\
\hline INTRODUCTION & المقدمة المقة \\
\hline MATERIALS AND METHODS & الأسلوب البحثى \\
\hline RESULTS AND DISCUSSION & النتائج ومناقشتها \\
\hline REFERENCES & المراجع \\
\hline ABSTRACT & الملخص \\
\hline INTRODUCTION & المقدمة المقة \\
\hline MATERIALS AND METHODS & 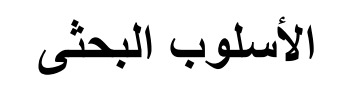 \\
\hline RESULTS AND DISCUSSION & النتائج ومناقشتها \\
\hline REFERENCES & المراجـع \\
\hline
\end{tabular}

